\title{
Abundância de Stomoxys calcitrans (Diptera: Muscidae) em diferentes subprodutos canavieiros ${ }^{1}$
}

\author{
Elaine C. Corrêa ${ }^{2 *}$, Augusto Cesar A. Ribas², Jairo Campos $^{3}$ e Antonio Thadeu M. Barros ${ }^{4}$
}

\begin{abstract}
Corrêa E.C., Ribas A.C.A., Campos J. \& Barros A.T.M. 2013. [Abundance of Stomoxys calcitrans (Diptera: Muscidae) in different sugar cane byproducts.] Abundância de Stomoxys calcitrans (Diptera: Muscidae) em diferentes subprodutos canavieiros. Pesquisa Veterinária Brasileira 33(11):1303-1308. Setor de Ecologia, Centro de Ciências Biológicas e da Saúde, Universidade Federal de Mato Grosso do Sul, Cidade Universitária, Cx. Postal 549, Campo Grande, MS 79070-900, Brazil. E-mail: elaineccorrea@yahoo.com.br

Increasing abundance of Stomoxys calcitrans has been observed in organic byproducts resulting from sugar cane alcohol production. This study aimed to evaluate the abundance of $S$. calcitrans in such byproducts. From January to December 2011, the abundance of $S$. calcitrans was monitored in four byproducts: sugar cane bagasse, mulch, filter cake (FC) and mulch plus vinasse (MV), in a sugar cane mill located at the municipality of Angélica, state of Mato Grosso do Sul, Brazil. Monthly, 20 emergence traps were placed in each substrate, remaining active for the following four weeks. A total of 4,049 specimens of S. calcitrans was caught during the whole period, representing $9.22 \%$ of total amount of collected flies. The four byproducts studied showed significant differences regarding the abundance of S. calcitrans, being higher in FC (67.20\%) and MV (29.19\%). Abundance peaks were observed in June (PV) and October (PV and TF). The highest productivity of S. calcitrans was observed in TF (55.8 flies $\left./ \mathrm{m}^{2}\right)$ and PV $\left(24.2 \mathrm{flies} / \mathrm{m}^{2}\right)$ and the average monthly production of $S$. calcitrans at the mill was estimated at 37,000 and 24 million, respectively. The high capacity of $S$. calcitrans breeding in sugar cane byproducts explains the recent population explosions of this species reported in cattle ranches around sugarcane mills.
\end{abstract}

INDEX TERMS: Stable fly, Stomoxys calcitrans, sugar cane mill, population explosion.

RESUMO.- Um aumento na abundância de Stomoxys calcitrans tem sido observado em áreas de produção sucroalcooleira devido aos subprodutos orgânicos resultantes desta atividade. 0 objetivo do presente estudo foi avaliar a abundância desta espécie em diferentes subprodutos da cana-de-açúcar. De janeiro a dezembro de 2011, a abundância de $S$. calcitrans foi monitorada em quatro subprodutos: bagaço, palha, torta de filtro (TF) e palha com vi-

\footnotetext{
${ }^{1}$ Recebido em 22 de junho de 2013.

Aceito para publicação em 30 de agosto de 2013.

${ }^{2}$ Setor de Ecologia, Centro de Ciências Biológicas e da Saúde, Universidade Federal de Mato Grosso do Sul (UFMS), Cidade Universitária, Cx. Postal 549, Campo Grande, MS 79070-900 Brasil. *Autor para correspondência: elaineccorrea@yahoo.com.br

${ }^{3}$ Unidade II, Faculdade de Ciências Biológicas e Ambientais, Universidade Federal da Grande Dourados (UFGD), Rodovia Dourados-Itahum, Km 12, Cx. Postal 322, Dourados, MS 79804-970, Brasil.

${ }^{4}$ Embrapa Gado de Corte, Av. Rádio Maia 830, Zona Rural, Campo Grande, MS 79106-550, Brasil.
}

nhaça (PV), em uma usina sucroalcooleira no município de Angélica, Mato Grosso do Sul, Brasil. Mensalmente, 20 armadilhas de emergência foram distribuídas em cada substrato, mantidas ativas por quatro semanas. Durante todo o período de estudo, 4.049 espécimes de $S$. calcitrans foram coletados nos diferentes substratos, representando 9,22\% do total de dípteros capturados nas armadilhas. Os quatro subprodutos amostrados apresentaram significativas diferenças em relação à abundância de $S$. calcitrans, sendo maior na TF $(67,20 \%)$ e na PV $(29,19 \%)$. Picos de abundância foram observados em junho (PV) e outubro (PV e TF). Maior produtividade de $S$. calcitrans foi observada na TF $\left(55,8\right.$ moscas $\left./ \mathrm{m}^{2}\right)$ e na PV $\left(24,2\right.$ moscas $\left./ \mathrm{m}^{2}\right)$, com produção média mensal de $S$. calcitrans na usina estimada em 37 mil e 24 milhões, respectivamente. A elevada capacidade de reprodução da S. calcitrans em subprodutos da usina explica as explosões populacionais desta espécie, ocorridas recentemente em fazendas pecuárias próximas a usinas sucroalcooleiras. 
TERMOS DE INDEXAÇÃO: Mosca-dos-estábulos, Stomoxys calcitrans, usina sucroalcooleira, explosão populacional.

\section{INTRODUÇÃO}

A mosca-dos-estábulos, Stomoxys calcitrans (L.) (Diptera: Muscidae), é uma importante praga de bovinos confinados e criados extensivamente (Hall et al. 1982, Taylor \& Berkebile 2011). Devido a seu hábito hematófago e o consequente incômodo causado aos animais, infestações por esta mosca podem afetar o ganho de peso e a produção de leite (Campbell et al. 2001), com perdas anuais superiores a US\$ 2 bilhões à bovinocultura nos Estados Unidos (Taylor et al. 2012) e US\$ 100 milhões no Brasil (Grisi et al. 2002).

As formas larvais de $S$. calcitrans se desenvolvem comumente em matéria orgânica em fermentação (Lysyk et al. 1999), principalmente contendo estrume e urina de bovinos (Skoda \& Thomas 1993). Além disso, são encontradas em substratos industriais, como fardos de feno (Hall et al. 1982), cama de frango (estrume de aves com restos vegetais) (Cook et al. 1999), silagem (Meyer \& Petersen 1983), biosólidos desidratados (bolo residual do tratamento de esgoto) (Doud et al. 2012), entre outros. 0 desenvolvimento reprodutivo de $S$. calcitrans em substratos efêmeros é influenciado pela umidade, temperatura, $\mathrm{pH}$ e competição interespecífica (Rasmussen \& Campbell 1981) e a abundância de adultos no ambiente está diretamente relacionada à disponibilidade de recursos para o desenvolvimento larval (Foil \& Hogsette 1994), condições microclimáticas do substrato e variáveis climáticas.

Explosões populacionais de $S$. calcitrans têm sido recentemente associadas à expansão da indústria sucroalcooleira em áreas de pecuária e aos subprodutos gerados em larga escala por essa atividade (Barros et al. 2010). Estes surtos, ocorridos principalmente nas regiões Centro-Oeste e Sudeste do Brasil, têm causado expressivos problemas à bovinocultura regional (Gomes 2009, Koller et al. 2009, Oda \& Arantes 2010). Durante os surtos, uma alta densidade dessa espécie tem sido observada, principalmente em bovinos e equinos, causando estresse e reduzindo a produtividade em fazendas próximas às usinas (Barros et al. 2010). Na tentativa de prevenir e controlar o problema, tanto na área canavieira como nas fazendas próximas, investimentos realizados por uma das usinas afetadas foram superiores a R $\$ 300$ mil em dois anos de ocorrência de surtos (informação pessoal, I. F. Silvério, departamento agrícola de usina sucroalcooleira).

De modo geral, os subprodutos da indústria sucroalcooleira são orgânicos e ricos em nutrientes, como cálcio, nitrogênio, potássio e enxofre (Vitti \& Luz 2008). Dependendo da forma como esses subprodutos são manejados e utilizados nas áreas de plantio, podem fornecer condições adequadas à sobrevivência e desenvolvimento de formas imaturas de moscas, particularmente Musca domestica (L.) (Buralli \& Guimarães 1985) e S. calcitrans (Nakano et al. 1973).

Considerando a importância das explosões populacionais ocorridas em áreas de produção canavieira, no presente estudo avaliamos a abundância de $S$. calcitrans em diferentes subprodutos de uma usina sucroalcooleira.

\section{MATERIAL E MÉTODOS}

\section{Área de estudo}

0 estudo de campo foi realizado de 07 /janeiro a 06/dezembro de 2011, em uma usina sucroalcooleira, localizada no município de Angélica (22 02' 28,22" S e 53 50’ 24, 48” W), Mato Grosso do Sul, Brasil.

Os principais subprodutos gerados pela usina incluíram: palha da cana (depositada no solo durante o processo de colheita), bagaço (material fibroso resultante do processo de extração do caldo da cana), cinza (oriunda da queima do bagaço para produção de energia), vinhaça (resíduo da destilação do caldo fermentado) e torta de filtro (sedimento da filtragem do caldo da cana). Entre abril e dezembro de 2011 foi gerado na referida usina um total de $1.438 .294 \mathrm{~m}^{3}$ de vinhaça e 85.359 toneladas de torta de filtro; os subprodutos bagaço e palha não foram contabilizados. A vinhaça produzida era armazenada em tanque e aplicada nas áreas de canavial próximas à usina. 0 armazenamento da torta de filtro era realizado em leiras depositadas no pátio de compostagem, com média de 15 a 18 leiras $(250-300 \mathrm{~m})$ produzidas a cada mês durante o período de safra.

\section{Armadilha de emergência}

Para determinar a abundância de Stomoxys calcitrans nos subprodutos foram utilizadas armadilhas de emergência, adaptadas a partir do modelo utilizado por Kunz et al. (1970).

As armadilhas de emergência eram constituídas por uma armação metálica piramidal $(45 \times 45 \times 45 \mathrm{~cm})$, com pinos (vergalhão de ferro de $16 \mathrm{~cm}$ ) nas arestas da base (para fixação nos substratos) e revestimento de tecido translúcido (organza de poliéster). 0 topo da armação metálica era formado por um anel, para encaixe de um funil plástico (parte superior de uma garrafa "pet" transparente de 2 litros), o qual foi conectado a um coletor, constituído por uma garrafa plástica transparente (pet) de 2 litros, para a captura de adultos recém-emergidos. A conexão entre funil e coletor foi feita utilizando-se uma tampa de garrafa pet, vazada, colada em um orifício na parte central do coletor (Fig.1). A estrutura utilizada facilitou a remoção e reposição dos coletores nas armadilhas.

A

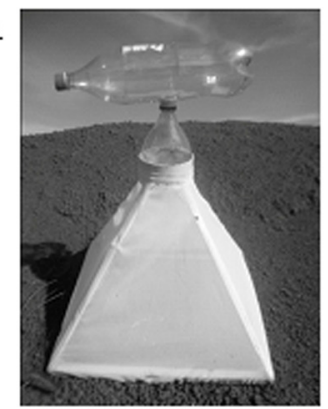

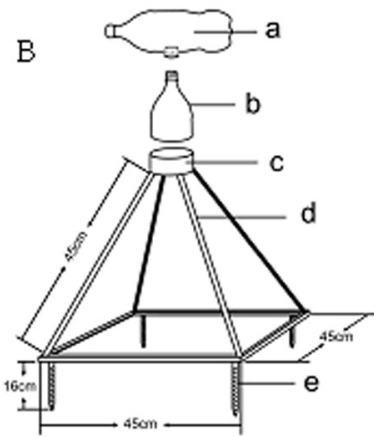

Fig.1. (A) Armadilha de emergência utilizada no monitoramento de Stomoxys calcitrans, (B) com detalhamento da estrutura: coletor (a), funil (b), anel de suporte (c), armação piramidal (d) e base com pinos de fixação no solo (e).

\section{Delineamento amostral}

Mensalmente, foram instaladas 20 armadilhas de emergência em cada substrato, permanecendo continuamente ativas durante quatro semanas no mesmo local. Em função da variação da disponibilidade dos subprodutos, os locais de instalação das armadilhas mudavam a cada mês nas suas respectivas áreas de armazenamento e/ou distribuição. Após a instalação das armadilhas, nenhuma prática de manejo foi realizada nos locais. 
Duas vezes por semana procedeu-se a coleta do material entomológico presente nas 80 armadilhas. Os insetos capturados nos coletores das armadilhas foram identificados no Laboratório de Entomologia da Universidade Federal da Grande Dourados, Dourados, Mato Grosso do Sul.

Durante todo o período, registros semanais referentes à temperatura no interior dos substratos foram obtidos através de um termômetro de solo $(20 \mathrm{~cm}$ de comprimento e $300 \mathrm{~mm}$ de sonda de medição), inserido próximo a cinco armadilhas sorteadas aleatoriamente.

\section{Análises estatísticas}

0 efeito dos subprodutos na abundância de $S$. calcitrans foi determinado utilizando-se um modelo geral linear misto (GLMM) com distribuição de Poisson. Esta distribuição é indicada para representar dados de contagem (Zuur et al. 2009) e tratar as datas das coletas e armadilhas como variáveis aleatórias que permitem melhor comportar as diferenças de abundância de $S$. calcitrans registradas para todo o período; não confundindo a variação temporal dos dados com as diferenças de abundância dos substratos, as quais constituíram uma variável fixa no modelo GLMM produzido (Bolker et al. 2008).

Um possível desvio na razão sexual de machos e fêmeas de $S$. calcitrans foi avaliado através de teste de Qui-quadrado usando a proporção de 1:1 como frequência esperada.

A produção potencial de $S$. calcitrans nos principais subprodutos foi obtida extrapolando-se o número médio de $S$. calcitrans nas 20 armadilhas/coleta (área total de $4,05 \mathrm{~m}^{2}$ ) durante todo o estudo, para as áreas ocupadas mensalmente pelos substratos na usina. A área de canavial com aplicação de vinhaça foi de aproximadamente $800 \mathrm{ha} / \mathrm{mês}$. Considerando uma faixa de $0,30 \mathrm{~m}$ de cada lado da base das leiras como adequada ao desenvolvimento larval (conforme constatado ao longo do estudo), a área da torta de filtro com potencial produção de moscas foi calculada em cerca de 0,27 ha/mês. A distribuição não homogênea de $S$. calcitrans nos substratos, confirmada por uma frequência relativamente baixa de armadilhas com esta espécie nas coletas, evidenciou que a produção desta mosca não ocorreu em toda a área coberta pelo substrato. A frequência média de armadilhas com $S$. calcitrans na palha com vinhaça $(12,23 \%)$ e na torta de filtro $(24,57 \%)$ foi usada na correção da estimativa da área total de produção da mosca nestes substratos.

Todos os testes estatísticos foram avaliados ao nível de significância de 0,05, utilizando a linguagem R (R CORE TEAM 2013) e o pacote lme4 (Bates et al. 2012) para construção dos modelos.

\section{RESULTADOS}

Durante todo o período de estudo, 4.049 espécimes de Stomoxys calcitrans foram coletados nos distintos subprodutos, representando 9,22\% do total de dípteros capturados nas armadilhas.

Destes, 4.029 espécimes foram sexados, sendo o número de fêmeas $(n=2.976)$ significativamente maior $\left(\chi^{2}=917,828, p<0.001\right)$ que o de machos $(n=1.053)$, evidenciando um desvio da razão sexual esperada (1:1), para aproximadamente 1:3. Este desvio na razão sexual foi observado em todos os subprodutos, sendo maior na torta de filtro (Fig.2a) e variou ao longo do tempo na torta e na palha com vinhaça (Fig.2b,c).

A temperatura no interior do substrato diferiu significativamente entre os subprodutos estudados. Torta de filtro, bagaço da cana, palha com vinhaça e palha apresentaram temperaturas médias de $35,45( \pm 5,82), 35,17( \pm 5,61)$, $21,14( \pm 4,94)$ e $18,80( \pm 5,36){ }^{\circ} \mathrm{C}$, respectivamente, durante todo o período.

Os quatro subprodutos foram significativamente diferentes quanto à abundância de $S$. calcitrans (Quadro 1). Os substratos com maior abundância dessa espécie foram torta de filtro $(n=2.721)$ e palha com vinhaça $(n=1.182)$

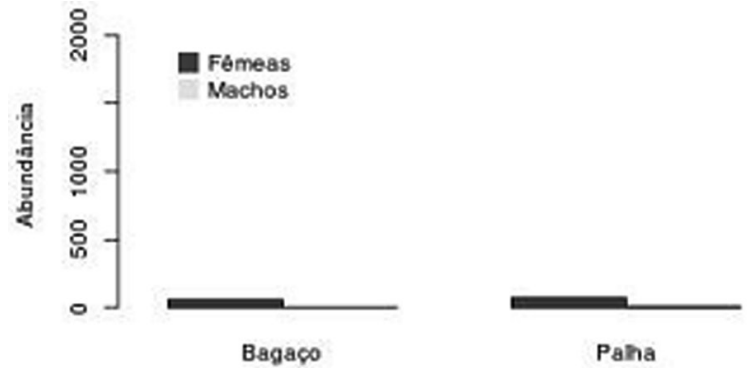

Palha com vinhaça

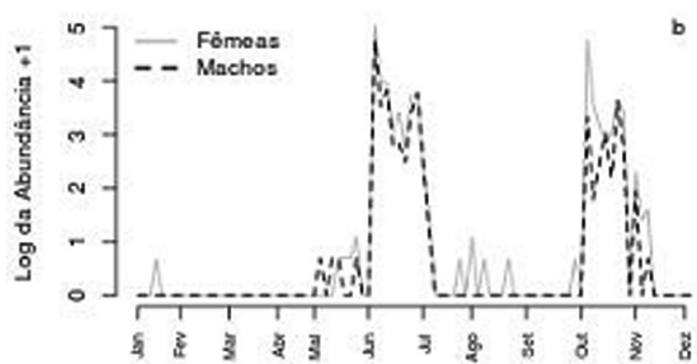

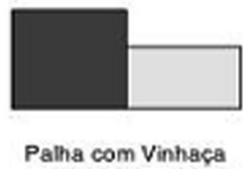

Palha com Vinhaca

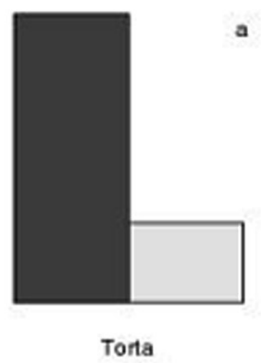

Torta

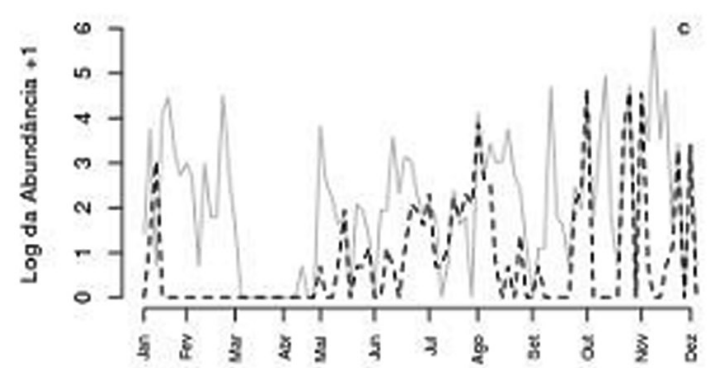

Fig.2. Abundância de Stomoxys calcitrans, por sexo, em subprodutos de usina sucroalcooleira, em Angélica, MS, de janeiro a dezembro de 2011: (a) abundância absoluta e (b) distribuição mensal nos principais substratos. 
Quadro 1. GLMM com distribuição de Poisson para: (a) abundância de Stomoxys calcitrans e (b) riqueza de espécies nos diferentes subprodutos de usina sucroalcooleira em Angélica, Mato Grosso do Sul

(a) GLMM da abundância

Efeito Aleatório

\begin{tabular}{lcccc}
\hline Fator & Variância & Desvio & & \\
Tempo & 3.645 & 1.909 & & \\
Armadilhas & 0.682 & 0.826 & & \\
Efeito Fixo & Estimativa & Erro & $\mathrm{Z}$ & $\mathrm{P}$ \\
Intercepto & -5.230 & 0.322 & -16.228 & $<0.001$ \\
Palha & 0.338 & 0.340 & 0.994 & 0.32 \\
Palha com vinhaça & 3.335 & 0.311 & 10.698 & $<0.001$ \\
Torta & 4.153 & 0.310 & 13.369 & $<0.001$
\end{tabular}

(Fig.3c,d), sendo significativamente menor sua abundância na palha $(n=83)$ e no bagaço $(n=63)$ (Fig.2a,b).

Com exceção das coletas realizadas em março e abril, S. calcitrans ocorreu em quase todas as semanas de coleta na torta de filtro ao longo do ano (Fig.3d). Os picos de abundância foram observados em junho e outubro na palha com vinhaça e em outubro, na torta de filtro (Fig.3c). Em ambos os substratos, a menor abundância foi observada no período de entressafra (janeiro-abril/2011) com capturas realizadas em substratos velhos, aumentando consideravelmente com o inicio da safra (abril/maio 2011) e a utilização de substratos recentes e potencialmente mais produtivos (Fig.3c,d).

A maior produtividade (produção por unidade de área) média de $S$. calcitrans nos substratos foi observada na torta de filtro $\left(55,8 \mathrm{moscas} / \mathrm{m}^{2}\right)$, seguida da palha com vinhaça

Bagaço

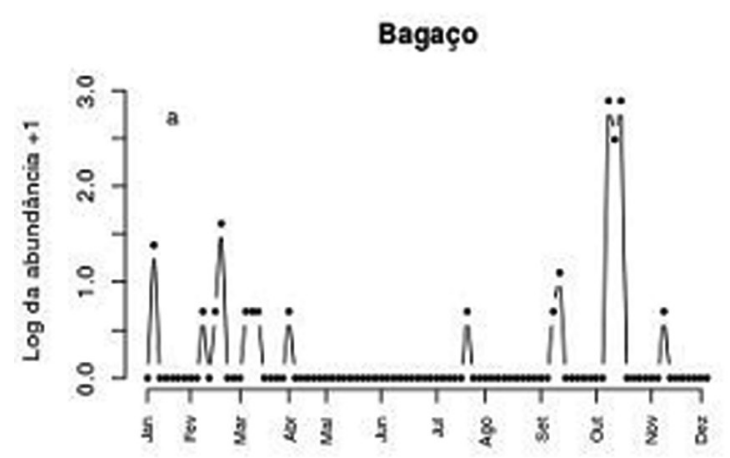

Palha com vinhaça

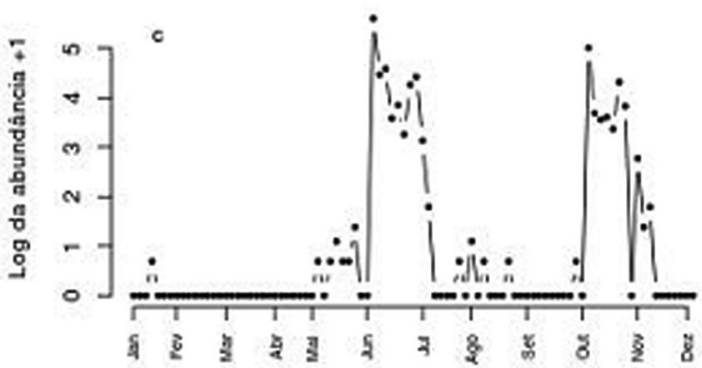

$\left(24,2\right.$ moscas $\left./ \mathrm{m}^{2}\right)$. Considerando uma produção total de $S$. calcitrans em função da área ocupada pelo substrato, em média, cerca de 37 mil moscas desta espécie podem ter emergido mensalmente da torta de filtro. Apesar da menor produtividade da palha com vinhaça, a extensa área coberta por este subproduto seria potencialmente capaz de produzir em torno de 24 milhões de $S$. calcitrans por mês, em apenas $12,23 \%$ da área mensalmente aplicada com vinhaça.

\section{DISCUSSÃO}

A elevada abundância populacional de Stomoxys calcitrans em torta de filtro e palha com vinhaça revelou sua estreita associação com subprodutos da indústria sucroalcooleira, confirmando o potencial destes ambientes no desenvolvimento larval desta espécie. De forma semelhante, Barros et al. (2010), em estudo sobre surto de S. calcitrans, verificaram um expressivo número de larvas de $S$. calcitrans em torta de filtro, assim como vários pupários de dípteros em canavial previamente irrigado com vinhaça. Vale salientar que a atual prática de aplicação de vinhaça no solo foi adotada para evitar a poluição de ambientes hídricos por descargas de vinhaça. Embora esse manejo reduza problemas com poluição ambiental, por outro lado contribui para a proliferação de moscas (Buralli \& Guimarães 1985), uma vez que a vinhaça utilizada no canavial forma um ambiente muito favorável ao desenvolvimento larval de $S$. calcitrans (Nakano et al. 1973).

Os picos de abundância observados na palha com vinhaça em junho e outubro, antecederam ou coincidiram com

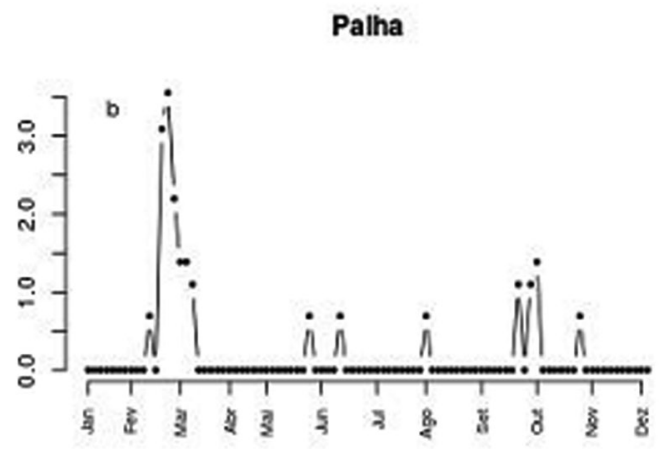

Fig.3. Abundância semanal de Stomoxys calcitrans, (a-d) em subprodutos de usina sucroalcooleira, em Angélica, MS, de janeiro a dezembro de 2011. 
surtos observados previamente ou durante o estudo, tanto em julho/agosto (Koller et al. 2009, Corrêa et al. 2012), como em outubro/novembro (Koller et al. 2009, Barros et al. 2010) na região de estudo. 0 pico de abundância observado na primavera (outubro) se assemelha ao pico de $S$. calcitrans observado em novembro/dezembro, em estudo sobre dinâmica populacional em Minas Gerais (Rodríguez-Batista et al. 2005). A favorabilidade desta época do ano à ocorrência de picos populacionais de $S$. calcitrans, aliada à grande extensão dos ambientes de reprodução e condições climáticas favoráveis, pode resultar na ocorrência de surtos, assim como observado na região estudada e em outras localidades do Estado.

A temperatura é uma variável ambiental determinante à taxa de desenvolvimento de moscas (Lysyk 1998), assim, é importante ressaltar que $S$. calcitrans se desenvolveu em substratos com temperaturas médias distintas $(18,8-$ $\left.35,5^{\circ} \mathrm{C}\right)$, sendo mais abundante na torta de filtro $\left(35,45^{\circ} \mathrm{C}\right)$ e na palha com vinhaça $\left(21,14^{\circ} \mathrm{C}\right)$. De forma semelhante, Lysyk (1998) observou maior sobrevivência de imaturos de $S$. calcitrans em temperaturas de $20-23^{\circ} \mathrm{C}$ e Berry et al. (1976) observaram o desenvolvimento de ovo a adulto em temperaturas superiores a $30^{\circ} \mathrm{C}$ em substratos com feno.

A abundância de fêmeas de $S$. calcitrans foi maior que a de machos adultos em todos os subprodutos da cana-de-açúcar. Tal resultado difere do encontrado por Taylor \& Berkebile (2011) ao avaliarem padrões espaciais e temporais de larvas de $S$. calcitrans em fardo de feno, nos quais foi encontrada uma razão sexual de 1:1. 0 desvio na razão sexual, com predomínio de fêmeas em torta de filtro e palha com vinhaça, pode favorecer a ocorrência de explosões populacionais, uma vez que a maior proporção de fêmeas tende a aumentar as taxas intrínsecas de crescimento da população, já que um maior número de fêmeas possibilita maior taxa de oviposição (Zump 1973).

A produção mensal de $S$. calcitrans na usina foi estimada em 37 mil e 24 milhões, em torta de filtro e palha com vinhaça, respectivamente, o que demonstra o elevado potencial destes substratos canavieiros como habitats de reprodução. Embora a produção de $S$. calcitrans tenha sido impressionante, sua produção média mensal de 55,8 e 24,2 moscas $/ \mathrm{m}^{2}$, respectivamente, foi muito inferior à encontrada no centro-oeste dos Estados Unidos, onde variou 1.632 a 19.600 moscas $/ \mathrm{m}^{2}$ de feno misturado a dejetos bovinos (Broce et al. 2005). Em última análise, a abundância de $S$. calcitrans no ambiente resultou não apenas do potencial de produção de adultos dos substratos, mas, também, da área coberta pelos sítios de reprodução por eles formados.

A elevada capacidade de produção massiva de $S$. calcitrans em subprodutos do processamento da cana-de-açúcar permite afirmar que esses substratos não apenas contribuem para a manutenção da população de S. calcitrans ao longo do ano, como visto em torta de filtro, mas predispõem à ocorrência de explosões populacionais, particularmente quando associadas a condições climáticas favoráveis.

Estudos científicos adicionais são necessários para identificar os fatores físico-químicos dos subprodutos canavieiros envolvidos na atração de adultos e posterior desenvolvimento larval de $S$. calcitrans nos referidos substratos.
Agradecimentos.- À Coordenação de Aperfeiçoamento de Pessoal de Nível Superior (CAPES), pela bolsa de estudo, e à Usina Angélica Agroenergia Ltda, pelo suporte financeiro e disponibilidade da infraestrutura. A Ademir Silva, Edi Carlos Silva e Isaias Fernando Silvério, pelo apoio em campo e Antonio Ferrari pelo apoio às atividades laboratoriais.

\section{REFERÊNCIAS}

Barros A.T.M., Koller W.W., Catto J.B. \& Soares C.O. 2010. Surtos por Stomoxys calcitrans em gado de corte no Mato Grosso do Sul. Pesq. Vet. Bras. 30(11):945-952.

Bates D., Maechler M. \& Bolker B. 2012. lme4: Linear mixed-effects models using S4 classes. R package version 0.999999-0. http://CRAN.R-project. org/package $=$ lme 4

Berry I.L., Foerster K.W. \& Ilcken E.H. 1976. Prediction model for development time of stable flies. Trans. Am. Soc. Agric. Eng. 19:123-127.

Bolker B.M., Brooks M.E., Clark C.J., Geange S.W., Poulsen J.R., Stevens M.H.H. \& White J.S.S. 2008. Generalized linear mixed models: a practical guide for ecology and evolution. Trends Ecol. Evol. 24(3):127-135.

Broce A.B., Hogsette J. \& Paisley S. 2005. Winter feeding sites of hay in round bales as major developmental sites of Stomoxys calcitrans (Diptera: Muscidae) in pastures in Spring and Summer. J. Econ. Entomol. 98(6):2307-2312.

Buralli G.M. \& Guimarães J.H. 1985. Controle de Musca domestica Linnaeus (Diptera, Muscidae) em área de manejo de vinhaça (Macatuba, São Paulo, Brasil). Revta Bras. Zool. 3(1):1-6.

Campbell J.B., Skoda S.R., Berkebile D.R., Boxler D.J., Thomas G.D., Adams D.C. \& Davis R. 2001. Effects of stable flies (Diptera: Muscidae) on weight gains of grazing yearling cattle. J. Econ. Entomol. 94(3):780-783.

Cook D.F., Dadour I.R. \& Keals N.J. 1999. Stable fly, house fly (Diptera: Muscidae), and other nuisance fly development in poultry litter associated with horticultural crop production. J. Econ. Entomol. 92(6):1352-1357.

Corrêa E.C., Gaona J.C. \& Barros A.T.M. 2012. Abundância de Stomoxys calcitrans (Diptera: Muscidae) associada com subprodutos de usina sucroalcooleira. Anais $29^{\circ}$ Congresso Brasileiro de Zoologia, Salvador, BA p.1075. (Resumo)

Doud C.W., Taylor D.B. \& Zurek L. 2012. Dewatered sewage biosolids provide a productive larval habitat for stable flies and house flies (Diptera: Muscidae). J. Med. Entomol. 49(2):286-292.

Foil L.D. \& Hogsette J.A. 1994. Biology and control of tabanids, stable flies and horn flies. Rev. Sci. Tech. Off. Int. Epizoot. 13(4):1125-1158.

Gomes R.A. 2009. Surtos de Stomoxys calcitrans (Diptera: Muscidae) em bovinos e eqüinos na região Noroeste de São Paulo/Brasil devido ao desequilíbrio ambiental. Engormix.com, Artigos Técnicos, Pecuária de Corte. Disponível em <http://pt.engormix.com/MA-pecuariacorte/saude/artigos/surtos-stomoxys-calcitrans-diptera_132.htm> Acesso em 9 fev. 2010.

Grisi L., Massard C.L., Moya Borja C.E. \& Perreira J.B. 2002. Impacto econômico das principais ectoparasitoses em bovinos no Brasil. Hora Vet. 125:8-10.

Guglielmone A.A., Volpogni M.M., Quaino O.R., Anziani O.S. \& Mangold A.J. 2004. Abundance of stable flies on heifers treated for control of horn flies with organophosphate impregnated ear tags. Med. Vet. Entomol. 18:10-13.

Hall R.D., Thomas G.D. \& Morgan C.E. 1982. Stable fly, Stomoxys calcitrans (L.), breeding in large round hay bales: Initial associations (Diptera: Muscidae). J. Kansas Entomol. Soc. 55(3):617-620.

Kunz S.E., Blume R.R., Hogan B.F. \& Matter J.J. 1970. Biological and ecological investigations of horn flies in Central Texas: Influence of time of manure deposition on ovoposition. J. Econ. Entomol. 63:920-923.

Koller W.W., Catto J.B., Bianchin I., Soares C.O., Paiva F., Tavares L.E.R. \& Graciolli G. 2009. Surtos da mosca-dos-estábulos, Stomoxys calcitrans, em Mato Grosso do Sul: novo problema para as cadeias produtivas da carne e sucroalcooleira? Documentos 175, Embrapa Gado de Corte, Campo Grande, MS. 31p. Disponível em <http://www.cnpgc.embrapa. br/publicaçoes/doc/doc_pdf/D0C175.pdf> Acesso em 31 mar. 2011. 
Lysyk T., Kalischuk-Tymensen L., Selinger L., Lancaster R., Wever L. \& Cheng K. 1999. Rearing stable fly larvae (Diptera: Muscidae) on an egg yolk medium. J. Med. Entomol. 36(3):382-388.

Lysyk T.J. 1998. Relationship between temperature and life-history parameters of Stomoxys calcitrans (Diptera: Muscidae). J. Med. Entomol. 35(2):107-119.

Meyer J.A. \& Petersen J.J. 1983. Characterization and seasonal distribution of breeding sites of stable flies and house flies (Diptera: Muscidae) on eastern Nebraska feedlot and dairies. J. Econ. Entomol. 76(1):103-108.

Nakano O., Paro Jr L.A. \& Camargo A.H. 1973. Controle químico de adultos e larvas da mosca doméstica. Biológico, São Paulo, 39:5-8.

Oda F.H. \& Arantes C.A. 2010. Surto populacional da mosca dos estábulos Stomoxys calcitrans Linnaeus, 1758 (Diptera: Muscidae) no município de Planalto, SP. Revta Agronegócios e Meio Ambiente 3(1):145-159.

R Core Team. 2013. R: A language and environment for statistical computing. R Foundation for Statistical Computing, Vienna, Austria. ISBN 3-900051-07-0, URL < http://www.R-project.org/>

Rasmussen R.L. \& Campbell J.B. 1981. Investigation of environmental factors and their relationship to populations of the stable fly, Stomoxys calcitrans (L.). Environ. Entomol. 10:798-800.

Rodríguez-Batista Z., Leite R.C., Oliveira P.R., Lopes C.M.L. \& Borges L.M.F. 2005. Populational dynamics of Stomoxys calcitrans L. (Diptera: Musci- dae) in three biocenosis, Minas Gerais, Brazil. Vet. Parasitol. 130:343346.

Skoda S.R. \& Thomas G.D. 1993. Breeding sites of stable flies and house flies, p.61-69. In: Thomas G.D. \& Skoda S.R. (Eds), Rural Flies in the Urban Environment. Res. Bull. 317, Institute of Agriculture and Natural Resources, University of Nebraska, Lincoln, NE.

Taylor D.B. \& Berkebile D.R. 2011. Phenology of stable fly (Diptera: Muscidae) larvae in round bale hay feeding sites in eastern Nebraska. Environ. Entomol. 40(2):184-193.

Taylor D.B., Moon R.D. \& Mark D.R. 2012. Economic impact of stable flies (Diptera: Muscidae) on dairy and beef cattle production. J. Med. Entomol. 49(1):198-209.

Vitti G.C. \& Luz P.H.C. 2008. Manejo e uso de fertilizantes para cana-de-açúcar, p.141-167. In: Marques M.O., Mutton M.A., Nogueira T.A.R., Tasso Jr L.C., Nogueira G.A. \& Bernardi J.H. (Eds), Tecnologias na Agroindústria Canavieira. FCAV, Jaboticabal, SP.

Zumpt F. 1973. The Stomoxyine Biting Flies of the World (Diptera: Muscidae): taxonomy, biology, economic importance and control measures. Gustav Fischer Verlag, Stuttgart. 175p.

Zuur A.F., Ieno E.N., Walker N.J., Saveliev A.A. \& Smith G.M. 2009. Mixed Effects Models and Extensions in Ecology with R. Springer, New York. $574 p$. 